\title{
Surrogate Endpoint: Alternative for Early Assessment of a Potential Treatment Effect
}

\section{R. Santosh Kumar* and Ganesh Sai Myneni}

GITAM Institute of Pharmacy, GITAM (Deemed to be University), Rushikonda, Visakhapatnam, Andhra Pradesh - 530045.

\begin{abstract}
The efficacy of health technologies, medicines and medical devices should be demonstrated in trails that evaluate final patient-relevant outcomes such as survival or morbidity. We provide a summary of the present use of surrogate end points in health care policy, discussing the case for and against their reviewing and adoption validation methods. Although the use of surrogates can be problematic, they can be validated and selected properly, offers important chances for more efficient clinical trials and faster access to new health technologies that benefit health care systems and patients. In early drug development studies, tumor response is often the true primary endpoint. Usually clinical trials are needed to show that it can be dependent upon to predict, or correlate with, clinical benefit in a context of use. Surrogate endpoints that have undergone this ample testing are called validated surrogate endpoints and these are accepted by the Food and Drug Administration as evidence of benefit. Choosing the right surrogate endpoint and proving that it can predict the intended clinical benefit, however, is not always straightforward. When a disease has been sufficiently studied, surrogate endpoints can measure the underlying cause of a dise ase (such as low thyroxine levels and hypothyroidism) or an effect that predicts the ultimate outcome (such as measuring diuresis, which is expected to improve symptoms of heart failure).
\end{abstract}

Keywords: Surrogate endpoint, Clinical trials, Oncology

Article Info: Received 17 June 2019; $\quad$ Review Completed 21 August 2019; Accepted 24 August 2019; $\quad$ Available online 30 August 2019

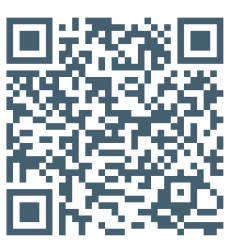

Cite this article as:

Santosh Kumar R, Sai Myneni G, Surrogate Endpoint: Alternative for Early Assessment of a Potential Treatment Effect, Journal of Drug Delivery and Therapeutics. 2019; 9(4-s):819-821 http://dx.doi.org/10.22270/jddt.v9i4-s.3371

*Address for Correspondence:

R. Santosh Kumar, GITAM Institute of Pharmacy, GITAM (Deemed to be University), Gandhinagar, Visakhapatnam530045, Andhra Pradesh, INDIA.

\section{Introduction:}

In the development of a pharmaceutical product, a clinical trial is the major part conducted for the demonstration of efficacy and safety of the product. The measurement used to assess the effect of the treatment is termed as endpoint. Endpoints used to measure clinical benefit, such as reduction of mortality rate and prolongation of survival time, are usually considered "true" endpoints because clinical benefits are the ultimate purpose of a medical treatment. However, the practicality and feasibility of measuring a "true" endpoint may be limited because of various reasons. Therefore, it is important to have some alternative measurement that would allow early assessment of a potential treatment effect.

A surrogate endpoint is a biomarker that coordinates very well with the activity and toxicity of the molecule. It is generally an acceptable endpoint for registration of a molecule with regulatory agencies. A biomarker qualifies as a surrogate endpoint for a clinical endpoint after controlled clinical trials which show a statistically and clinically significant correlation between the two. Surrogate endpoints must reliably speculate both the safety and efficacy associated with a pharmacological agent.

Surrogate endpoints are sometimes obtained from studies evaluating the natural history of the epidemiology. There are few examples of surrogate endpoint such as blood pressure is an accepted surrogate endpoint for anti-hypertensive agents as it predicts cardiovascular disease, heart failure, stroke and kidney failure [1]. For example, for several classes of agents bone mineral density has shown to have a good correlation with fracture rates. However, this has not turned out to be the case for fluoride [2].

The limitations of "true" endpoints prompted the use of surrogate endpoints. Surrogate endpoints that are considered to relate clinically important outcome but does not in itself measure a clinical benefit.

This surrogate plays a particularly important role in the early development of a pharmaceutical product, when surrogate endpoints can be used to demonstrate if the drug has any pharmacological effect, as postulated by the in vitro model or in preclinical in vivo studies. 
A surrogate is mainly useful if it is easily measured and highly correlated with the true endpoint. Often, 'true' endpoint is one with the clinical importance to the patient. For example, a major clinical outcome or mortality, while a surrogate is one biologically closer to the process of disease, for example, ejection fraction. Use of the surrogate can often lead to dramatic reductions in sample size and much shorter studies than use of the true endpoint [3]

Atkinson [4] has compiled a table of biomarkers which have found varying degrees of clinical utility. Tables 1

Table 1: Biomarkers/surrogate endpoints that have aided drug development

\begin{tabular}{lll}
\hline Biomarker / surrogate endpoint & Type of drug & Clinical endpoint \\
\hline Blood pressure & Antihypertensive Agents & Stroke, atherosclerosis. Heart failure \\
Cholesterol & LDL lowering statins & Coronary artery disease, heart attacks \\
Viral RNA & Antiretroviral agents & Survival, decrease in infections \\
HbA $_{1 c}$ Glucose & Antidiabetic agents & Diabetic neuropathy \\
$\mathrm{CD}_{4}+\mathrm{T}$ cells & Antiretroviral agents, cytokines & Sustained reduction in viral RNA \\
Intraocular pressure & Antiglaucoma agents & Preservation of peripheral vision \\
Bone mineral density (BMD) & Antiosteoporotic agents & Fracture rate \\
MRI (Magnetic Resonance Imaging) Scans & Agents for treatment of Multiple & Decrease in rate of progression disease \\
& Sclerosis (MS) & (check PDR) \\
CT (Computed tomography) & Anticancer agents & Survival \\
Scans for tumor size & &
\end{tabular}

\section{Surrogate Endpoint related to Oncology:}

Surrogate endpoint in oncology is widespread and increasing. The strength of association between the surrogates used and clinically meaningful outcomes is often weak or unknown. Attempts to validate surrogates are rarely undertaken. When this is done, validation relies on a fraction of available data, and often concludes that the surrogate is poor. Alternatively, if a drug fails to improve quality of life or overall survival, market authorization is rarely revoked [5]

The most frequently used surrogates are response rate; a set of criteria distinguishing tumor shrinkage; and time to event endpoints, such as progression-free survival (PFS) or recurrence-free survival (RFS). Progression-free survival and Recurrence-free survival are composite endpoints where an event is explained as either growth of tumor beyond an arbitrary threshold (progression) or detectable recurrence of disease, or death. While there is debate as to whether PFS is intrinsically meaningful [6] since patients do not feel when they cross the arbitrary threshold of 'progression,' we believe that PFS is, strictly speaking, a surrogate.

\section{Surrogate endpoint related to cardiovascular disease:}

A commonly used example in the cardiovascular disease is cholesterol. While elevated cholesterol levels increase the cause of heart disease, the relationship is not linear - many people with normal cholesterol develop heart disease, and many with high cholesterol do not. A clinical trial may show that a drug effective in reducing cholesterol, without showing directly that it prevents death. Proof of its efficaciousness in reducing cardiovascular disease was only presented five years after its original introduction, and then only for secondary prevention [7]

\section{Are surrogate endpoints important for medical product development? [8]}

When a surrogate endpoint clearly speculates a beneficial effect through appropriate studies, its use commonly allows for more efficient drug development programs. For example, many clinical trials, using a range of different blood pressure lowering medications, have demonstrated that reducing systolic blood pressure reduced the risk of stroke. Hence, quantification of reduction, surrogate endpoint of systolic blood pressure can stand in for the clinical outcome of stroke, and clinical trials targeting the reduction of risk of stroke that can be conducted more rapidly in smaller populations using this validated surrogate endpoint.

$>$ Why use a surrogate endpoint?

- Faster and easier to study,

- e.g., Cholesterol or Blood Pressure vs. Stroke, Survival, Myocardial infarction.

- Faster drug development,

- Cheaper,

- Proving effect on direct endpoint may not be practicable.

- Very low event rates - use of the surrogate in common clinical practice may make definitive trial seem unethical [9].

\section{Examples of Surrogate Endpoints: [9]}

- Hypertension - arterial blood pressure: surrogate for Cerebrovascular accident, Myocardial Infarction, heart failure

- Hypercholesterolemia -surrogate for atherosclerotic disease is basing on cholesterol levels.

- Human Immunodeficiency Virus (HIV) - CD4 count or viral load: surrogate for complications of HIV

- Glaucoma - surrogate for loss of vision is intraocular pressure.

- Diabetes Mellitus - blood glucose / haemoglobin A1c: surrogate for complications.

\section{Limitations of using Surrogate endpoint:}

On yielding potentially important advantages, the use of surrogate endpoints, sometimes can show inappropriate results and thus causes problems. The two principal concerns on introduction of any proposed surrogate variable are it may not be true estimation of clinical outcome of 
interest. For example, surrogate endpoint may measure the treatment activity associated with one of a specific pharmacological mechanism, but it may not provide exactly complete information on the range of actions and ultimate effects of the treatment whether it is positive or negative. There have been many examples where treatments showing a highly positive effect on a proposed surrogate have ultimately been shown to be detrimental to the subjects of clinical outcome; conversely, there are cases of treatments conferring clinical benefit without measurable effect on proposed surrogates [10]. Some of the cancer treatments provide a positive effect in a treatment in surrogate endpoint, but a detrimental effect on a patient health. In this case, although a treatment may achieve a certain degree of tumor shrinkage, the toxicity of the drug itself may in fact worsen a patient's general health and thus shorten survival time.

\section{Regulatory Requirements:}

The Food and Drug Administration (FDA) must balance engaging needs in its approach to assess biomarkers, and it must do so with limited resources. On one hand, biomarkers are viewed as a route to reduce the cost and time required to develop effective new drugs, devices, and biologics to address chronic diseases; on the other hand, patients and consumers must be protected from risks associated with biomarker use. The perceptible efficiency dichotomy also applies to the use of biomarkers in health claims for foods, which the Food and Drug Administration regulates under a different framework than is applied to biologics, drugs and devices [11],

\section{Validating a Surrogate Endpoint}

- $\quad$ For a surrogate to be useful, the relationship between the surrogate and the "direct" endpoint must be strongly established. Simple correlations, no matter how strong, are not enough.

- The ideal method is the analyses of many studies of known effective drugs, which evaluated both the direct and surrogate endpoints, in order to establish (and quantitate) the relationship.

- Very difficult to identify what type and quantity of data are sufficient to adequately validate a surrogate for use as a primary endpoint in a Phase 3 trial.

- Once proved, a surrogate may be useful for future studies of medicines, particularly those with same mechanism of action.

\section{Conclusion:}

Post-marketing studies, planned to protect drugs have meaningful benefits, are often not performed. The potential for surrogate end points to have an impact on health care policy and the consequent diffusion of technologies into practice is illustrated by the fact that the primary outcome of more than $40 \%$ of pivotal trails used as the basis for approval of new indications is a surrogate that aims to substitute for and predict a final patient - relevant outcome.. The strength of association between the surrogates used and clinically meaningful outcomes are often unknown or weak. Attempts to validate surrogates are rarely undertaken. In both the cases surrogates must be used only when continuing studies examining hard endpoints have been fully enrolled. Relying on surrogates rather than final patient relevant outcomes increases the uncertainty when making decisions about licensing and coverage of health care technologies. The use of appropriately validated surrogate end points, however, provides an unmissable opportunity to speed up access to innovative technologies that offer important benefits for patients and health care systems and to improve efficiency within the research and development environment.

\section{References:}

1) A.V. Chobanian, The influence of hypertension and other hemodynamic factors in atherogenesis, Prog.Cardiovasc. Dis. 26 (1983), 177-196.

2) B.L. Riggs, S.F. Hodgson, W.M. O’Fallon, E.Y.S. Chao, H.W. Wahner et al., Effect of fluoride treatment on the fracture rate in postmenopausal women with osteoporosis, N. Engl. J. Med. 322 (1990), 802-809.

3) Wittes J, Lakatos E, Probstfield J, Surrogate endpoints in clinical trials: cardiovascular diseases. Stat Med., 1989 Apr:8(4):415-25.

4) A.J. Atkinson Jr., Physiological and laboratory markers of drug effect, in: Principles of Clinical Pharmacology, A.J. Atkinson Jr, C.E. Daniels, R.L. Dedrick, C. Grudzinskas and S.P. Markey, eds, New York: Academic, 2001.

5) Surrogate endpoints in oncology, Robert Kemp and Vinay Prasad.; BMC., 2017.

6) Booth CM, Eisenhauer EA. Progression-free survival: meaningful or simply measurable? J Clin Oncol. 2012. 30:1030-3.

7) Pedersen TR, Olsson AG, Faergeman 0, et al.. "Lipoprotein changes and reduction in the incidence of major coronary heart disease events in the Scandinavian Simvastatin Survival Study (4S)". Circulation. 97 (15) (1998): 1453-1460.

8) https://www.fda.gov/drugs/developmentresources/surrogate-endpoint-resources-drug-and-biologicdevelopment

9) Clinical Trial Endpoints, Eugene J. Sullivan, MD FCCP, Food and Drug Administration, https://www.fda.gov/media/84987.

10) ICH Harmonized Tripartite Guideline; Statistical Principles of Clinical Trials, S2.26, 1998

11) Perspectives on Biomarker and Surrogate Endpoint Evaluation, Douglas Balentine, Thomas Fleming, Philip Greenland, James Mayne; https://www.ncbi.nlm.nih.gov/books/NBK209574/ 